\title{
LA SENDA MINERA O CÓMO CONECTAR HISTORIA, PATRIMONIO Y DESARROLLO RURAL
}

\author{
The Mining Path or How To Connect History, Heritage \\ and Rural Development
}

\section{O caminho da mineração ou como conectar história, patrimônio e desenvolvimento rural}

\author{
Dr. Francisco M. BALADO INSUNZA \\ Investigador Dpto. H. ${ }^{\text {a }}$ Contemporánea UNED \\ Coordinador de Proyectos y Extensión Universitaria UNED Ponferrada \\ fbalado@ponferrada.uned.es
}

Fecha de recepción: 29/07/2020

Fecha de aceptación: 15/11/2020

RESUMEN: La actividad minera ha capitalizado la imagen histórica de El Bierzo y Laciana durante el último siglo. Fue el motor de su expansión y del devenir económico que ha forjado su realidad social durante décadas y condicionado la actual.

Los vestigios que existen hoy de esa actividad minera e industrial se presentan como una de las referencias históricas contemporáneas del Bierzo y Laciana, quizás la más importante y, sin duda, deben ser considerados como uno de los elementos esenciales de su identidad colectiva y, en consecuencia, patrimonio colectivo susceptible de ser preservado y puesto en valor.

Este patrimonio se presenta como un valor integral del territorio, un nexo entre los diferentes paisajes originados que, uniendo lo industrial y lo natural, se identifican en El Bierzo y Laciana, pudiendo establecerse puntos de contacto que pueden conformar un discurso coherente con una sólida base histórica, cultural y, sobre 
todo, en términos actuales, integradora desde una perspectiva social y económica, de todo el espacio delimitado por la actividad minera de la zona.

Dentro de las líneas de actuación que se pueden desarrollar combinando los elementos apuntados, está la de construir una ruta que una todos los municipios mineros de estas comarcas, identificando sus recursos patrimoniales individuales, subrayando sus singularidades de modo que se pueda ir formando una red que conecte todos los polos, complementariamente entre sí. De esta manera, todos los recursos, sin solaparse, se podrían incorporar a La Senda Minera, proyecto que recoge dos ideas clave: la de avance y progreso (ruta, camino, senda) y la de una minería, la de carbón y la de hierro, que nos recuerda la historia reciente y se proyecta, patrimonialmente, hacia el futuro como base del desarrollo de territorios especialmente desfavorecidos y atacados por la crisis y la despoblación.

Palabras clave: historia minera; vestigios patrimoniales; desarrollo rural; senda minera.

ABSTRACT: Mining activity has capitalized on the historical image of Bierzo and Laciana during the last century. It was the engine of its expansion and economic development that has forged its social reality for decades and conditioned the current one.

The vestiges that exist today of this mining and industrial activity are presented as one of the contemporary historical references of the Bierzo and Laciana, perhaps the most important and, without a doubt, should be considered as one of the essential elements of their collective identity and, in Consequently, collective heritage capable of being preserved and valued.

This heritage is presented as an integral value of the territory, a nexus between the different originated landscapes that, uniting the industrial and the natural, are identified in El Bierzo and Laciana, being able to establish points of contact that can form a coherent discourse with a solid base historical, cultural and, above all, in current terms, integrating from a social and economic perspective, of all the space delimited by the mining activity in the area.

Among the lines of action that can be developed by combining the elements mentioned, is to build a route that unites all the mining municipalities of these regions, identifying their individual heritage resources, highlighting their singularities so that a network can be formed that connect all the poles, complementary to each other. In this way, all the resources, without overlapping, could be incorporated into La Senda Minera, a project that includes two key ideas: that of advancement and progress (route, path, path) and that of a mining, that of coal and that of iron, which reminds us of recent history and is projected, patrimonially, into the future as the basis for the development of especially disadvantaged territories and attacked by crisis and depopulation.

Key words: mining history; heritage vestiges; rural development; mining path. 
FRANCISCO M. BALADO INSUNZA

LA SENDA MINERA O CÓMO CONECTAR HISTORIA, PATRIMONIO Y DESARROLLO RURAL

RESUMO: A atividade mineira capitalizou a imagem histórica de Bierzo e Laciana durante o século passado. Foi o motor de expansão e desenvolvimento econômico que forjou sua realidade social durante décadas e condicionou a atual. Os vestígios que hoje existem desta actividade mineira e industrial apresentam-se como uma das referências históricas contemporâneas de Bierzo e Laciana, talvez a mais importante e, sem dúvida, deve ser considerada como um dos elementos essenciais da sua identidade colectiva e, na Conseqüentemente, patrimônio coletivo passível de ser preservado e valorizado. Este património apresenta-se como um valor integrante do território, um nexo entre as diferentes paisagens originadas que, unindo o industrial e o natural, se identificam em El Bierzo e Laciana, podendo estabelecer pontos de contacto que possam constituir um discurso coerente com uma base sólida histórico, cultural e, sobretudo, em termos atuais, integrando do ponto de vista social e econômico, todo o espaço delimitado pela atividade mineira na área.

Entre as linhas de ação que podem ser desenvolvidas a partir da combinação dos elementos citados, está a construção de um roteiro que une todos os municípios mineiros dessas regiões, identificando seus recursos patrimoniais individuais, destacando suas singularidades para que se forme uma rede que conectar todos os pólos, complementares uns aos outros. Desta forma, todos os recursos, sem sobreposição, poderiam ser incorporados em La Senda Minera, um projeto que inclui duas ideias-chave: a de avanço e progresso (rota, caminho, caminho) e a de uma mineração, a de carvão e a de ferro, que nos faz lembrar a história recente e se projeta, patrimonialmente, no futuro como base para o desenvolvimento de territórios particularmente desfavorecidos e atacados pela crise e pelo despovoamento.

Palavras chave: história da mineração; vestígios do patrimônio; desenvolvimento rural; trilha da mineração.

\section{INTRODUCCIÓN}

La historia reciente de El Bierzo (y de Laciana) ${ }^{1}$ se halla inexcusablemente asociada a la minería, a la industria vinculada a la actividad extractiva y al ferrocarril que recorría las cuencas del occidente leonés, vertebrándolas en torno a la propia minería y su industria. Las comarcas de El Bierzo y de Laciana, situadas en el noroeste de la provincia de León y, por ello, en el extremo más noroccidental de la Comunidad Autónoma de Castilla y León, territorio periférico, con difíciles comunicaciones y colindantes con las Comunidades de Galicia y Asturias, han sido durante más de cien años uno de los polos de extracción carbonífera más importantes de la Península Ibérica (Rubio, 2012).

1. Las referencias hechas a la comarca del Bierzo incluyen la de Laciana y, en alguna medida, pequeña, la de otras comarcas como La Cepeda, que, a los efectos de este proyecto, conforman una unidad de acción incuestionable. 
La actividad minera ha capitalizado la imagen histórica de El Bierzo durante el último siglo. Fue el motor de su expansión y del devenir económico que forjó su realidad social hasta la actualidad.

Estos territorios mineros se han configurado como espacios funcionales de monocultivo productivo y, en un contexto histórico muy determinado, el del corto siglo XX (Balado, 2018), espacios generadores de la energía necesaria para acometer los importantes procesos de transformación que ha vivido España y que supusieron «el desmantelamiento del tejido productivo tradicional y afectaron a espacios de singular valor paisajístico y medioambiental, generando una muy especial "herencia» transgeneracional» (Rubio et al., 2012, p. 271).

Los vestigios que, de esa actividad minera e industrial, han llegado al tiempo actual, se presentan como una de las referencias históricas contemporáneas de El Bierzo, quizás la más importante y, sin duda, deben ser considerados como uno de los elementos esenciales de su identidad colectiva y, en consecuencia, patrimonio colectivo susceptible de ser preservado y puesto en valor.

Además, contemplando ese pasado desde nuestra realidad actual y proyectándolo hacia el futuro, el patrimonio minero e industrial de estas comarcas, con elementos y alcance distinto, en muchos casos singulares y, en todos, muy estimable, supone hoy, extinguida la actividad extractiva, una oportunidad y, en términos económicos, un recurso. Por otra parte, este patrimonio se presenta como un valor integral del territorio, un nexo entre los diferentes paisajes originados que, uniendo lo industrial y lo natural, se identifican en El Bierzo, pudiendo establecerse puntos de contacto que pueden conformar un discurso coherente con una sólida base histórica, cultural y, sobre todo, en términos actuales, integradora desde una perspectiva social y económica, de todo el espacio delimitado por la actividad minera de la zona.

Dentro de las líneas de actuación que se pueden desarrollar, combinando los elementos apuntados, está la de construir una ruta que una todos los municipios mineros de estas comarcas, identificando sus recursos patrimoniales individuales y, subrayando sus singularidades. La idea consiste en ir formando una red que conecte todos los polos, complementariamente entre sí, de manera que, sin solaparse, se incorporen a un proyecto global que hemos denominado La Senda Minera, denominación que recoge dos ideas clave: la de avance y progreso (ruta, camino, senda) y la minería que nos recuerda la esencia del proyecto, en todos sus términos.

Hay que decir que no existen muchos trabajos que traten de cimentar teóricamente el proyecto que se presenta en esta contribución. Se trata, por tanto, de una propuesta que se inserta en la Cátedra de Turismo Sostenible y Desarrollo Local (CTS y DL) proyecto del Consorcio Universitario del Centro Asociado a la UNED en Ponferrada ${ }^{2}$. El objeto de esta Cátedra no es otro que servir de catalizador de

2. http://catedraturismosostenible.es/ 
iniciativas que, usando sus recursos propios, patrimoniales o naturales, sirvan para generar actividad económica en territorios, como los que son objeto de la propuesta, especialmente afectados por los procesos de descarbonización (Calvo, Vega y Vázquez, 2020, 2).

La Senda Minera constituye una idea práctica para la valorización de sus vestigios mineros e industriales, la utilización sostenible de los recursos, la contribución a generar actividad económica y, como consecuencia de ello, la dinamización de estos territorios contribuyendo a su recuperación y fijación de población.

\section{LA CONSERVACIÓN, PROTECCIÓN Y PUESTA EN VALOR DEL PATRIMONIO MINERO- INDUSTRIAL. BASE NORMATIVA, CONCEPTUAL E HISTÓRICA}

El marco normativo general

Al profundizar en el concepto de Economía del Patrimonio Cultural, es decir, aquella que considera que los bienes del patrimonio cultural deben dejarse de percibir como una carga para considerarse un recurso capaz de generar desarrollo y cohesión social, se parte de la idea de que el patrimonio minero está constituido por estructuras inmuebles, objetos muebles, documentos y elementos inmateriales, o intangibles, vinculados con actividades mineras del pasado, a los que un grupo social, más o menos amplio, atribuye valores históricos, culturales o sociales (Puche Riart, 1994; Féraud, 2001; Mazadiego Martínez, 2003).

Tal idea se adapta al patrimonio derivado de la actividad tras la Revolución Industrial, la protección, en términos de políticas públicas, de paisajes mineros e industriales no es una novedad. En el plano internacional, este concepto se ha desarrollado desde la creación en 1978 del Comité Internacional para la Conservación del Patrimonio Industrial (TICCIH), creado en el año 1978 durante la III Conferencia Internacional sobre la Conservación de Monumentos Industriales que se celebró en Suecia. Implantado en más de cuarenta países, su objetivo esencial es promover la cooperación internacional para identificar, preservar, conservar, investigar, documentar y revalorizar el patrimonio industrial.

El Convenio Europeo del Paisaje (Florencia, 20 de octubre de 2000) auspiciado por el Consejo de Europa y ratificado por España el 26 de noviembre de 2007, constituye un punto de referencia para la consideración del espacio minero como paisaje cultural. La Estrategia Territorial Europea (ETE) incluye los paisajes culturales como integrantes del patrimonio cultural de la Unión Europea y los considera un factor económico de importancia creciente para el desarrollo sostenible.

En España no será hasta el siglo XXI cuando se comience a tomar verdadera conciencia pública de la necesidad de conservar y proteger el patrimonio industrial y, en concreto, el patrimonio derivado de la actividad minera. A este respecto constituyó todo un hito la denominada Carta de El Bierzo para la conservación del 
Patrimonio Industrial Minero 3 que en 2008 constituyó «un ejercicio de reflexión sobre los valores culturales propios de los bienes vinculados a la explotación minera de época industrial, presentando asimismo una propuesta metodológica para su preservación y disfrute público». La Carta de El Bierzo fue una de las guías que orientaron el Plan Nacional de Patrimonio Industrial que, desde 2011, supone el instrumento de referencia para la gestión de este patrimonio. Su necesidad se justificaba, entre otras razones, "por la necesidad de protección y conservación de un patrimonio que, por su propia especificidad, presenta un rápido deterioro y está expuesto a desaparecer ${ }^{4}$. Este patrimonio, precario, muy numeroso y disperso, sin duda, singular pero muy desprotegido legal, administrativa y socialmente, precisaba un plan general de actuación y eso ha intentado ser el Plan Nacional de $2011^{5}$.

En orden territorial descendente, en la Comunidad Autónoma de Castilla y León, administración competente en el ámbito industrial y, también, en el patrimonial ha adoptado medidas importantes. No se trata ahora de desarrollar aquí todas ellas, pero baste citar, a modo indicativo, las incorporadas al Plan PAHIS 2020 de intervención en el Patrimonio Cultural, cuyo objetivo final es la puesta en valor en este patrimonio industrial complejo ${ }^{6}$. Significativamente el Plan PAHIS recoge las ideas de valorización, preservación y utilización de los recursos patrimoniales como elementos para generar actividad económica en el territorio.

\section{El marco histórico general}

La actividad minera e industrial tradicional surgida con la revolución industrial, tecnológica, económica y social llegó a partir de la segunda mitad del siglo XIX a estas comarcas periféricas. Fue el progreso del ferrocarril, unido a las primeras explotaciones carboníferas las que atrajeron progresivamente el interés de grupos de los primeros capitalistas que fijaron su atención en estos territorios buscando nuevos yacimientos, reservas de materias primas y fuentes de energía,

3. Se trata de un documento elaborado por los técnicos del Instituto del Patrimonio Cultural de España. Fue presentado en unas jornadas técnicas celebradas en Ponferrada durante el mes de octubre de 2007, organizadas por el Instituto del Patrimonio Cultural de España y la Fundación Estatal Ciudad de la Energía, con la colaboración de la Junta de Castilla y León. En ellas estuvieron representadas todas las Comunidades Autónomas, a través de técnicos especializados en Patrimonio Industrial. En las conclusiones de aquellas jornadas, los intervinientes consensuaron un texto a modo de Manifiesto o Carta que fue elevado al Consejo de Patrimonio Histórico del 27 de junio de 2008, siendo aprobado por el mismo.

Véase https://ipce.culturaydeporte.gob.es/dam/jcr:9150e4a0-fb5b-40f9-8b42-df8f70187f27/cartadel-bierzo-layout1. pdf. Consultada el 10/05/2020.

4. Carta de El Bierzo, preámbulo.

5. http://www.culturaydeporte.gob.es/planes-nacionales/planes-nacionales/patrimonio-industrial. html. Consultada el 10/05/2020.

6. https://patrimoniocultural.jcyl.es/web/jcyl/PatrimonioCultural/es/Plantilla100Detalle/ 1284180255460/Publicacion/1284689348817/Redacción. Consultada el 10/05/2020. 
imprescindibles para la consolidación de las incipientes empresas industriales del tercio norte peninsular.

Esto se inscribe en la tesis de que el análisis económico general de la España de la Restauración (1875-1923) resalta los profundos cambios que comienzan a percibirse en las denominadas "constantes estructurales básicas" de la historia económica contemporánea de España (Forcadell, 1996, citado en Balado, 2018). En este sentido, José Luis García Delgado en su todavía imprescindible obra: La formación de la sociedad capitalista en España 1914-1920 fija el comienzo de la modernización de la economía española en el primer tercio del siglo XX, en un sentido global (citado en Balado, 2018, p.). Para este autor, crecimiento y cambio estructural, nítidos factores modernizadores, serían los dos rasgos más característicos del reinado de Alfonso XIII en términos económicos, sin obviar elementos de freno y conflicto inherentes a ese proceso que hicieron que el apreciable crecimiento de la economía española a lo largo del reinado de Alfonso XIII fuese insuficiente para recortar sustancialmente el atraso previo.

En este contexto, en El Bierzo y Laciana comienza lo que hemos denominado el "corto" siglo XX (1918-1993), definido como «un tiempo de grandes mutaciones sociales y económicas, de fuerte industrialización, de convulsiones políticas, de guerra y penalidades, de represión, de odios y venganzas personales teñidas de políticas, de diferentes e importantes flujos migratorios (de entrada y de salida), de desarrollo fulgurante, de cambios estructurales en los sistemas productivos de la comarca» (Balado 2018, pp. 21-22).

En este resumen, la industria, fundamentalmente extractiva, con claro empuje antes de la guerra civil y con auge extraordinario en los años inmediatamente posteriores a ella, fue la clave de bóveda que condicionó el proceso histórico de estas comarcas durante las décadas centrales del siglo XX. Su declive, a partir de la década de los setenta y, sobre todo de los ochenta del referido siglo, supuso el principio del fin de la viabilidad de la industria carbonífera con un hecho que supuso el paradigma del proceso que se producía: la primera quiebra de la empresa Minero-Siderúrgica de Ponferrada en 1993.

Este proceso de creación, auge y declive industrial, pero también social y medioambiental, de poco más de un siglo en términos contextuales y, con mayor precisión, de algo menos de ochenta años, ha ido, con el paso del tiempo, dejando pruebas materiales e inmateriales de su existencia, vestigios que esos procesos ya concluidos nos han llegado como tales, como elementos relevantes del proceso histórico y que nosotros, generaciones subsiguientes en el recorrido temporal, nos corresponde decidir sobre su propia existencia y continuidad.

En este sentido, utilizando los criterios normativos indicados en la introducción y la propia dinámica metodológica en cuanto a su conservación y gestión, se pueden y, en muchos casos, se deben ir incorporando al conjunto de bienes patrimoniales de una comunidad, en este caso, de las zonas mineras del oeste de la provincia de León, desde la Cepeda y El Bierzo hasta el valle de Laciana. La razón para su conservación reside en que se trata de pruebas de la actividad antrópica 
que se desarrolló en el contexto del monocultivo minero e industrial berciano y lacianiego, desde mediados del siglo XIX y hasta, en términos absolutos, $2018^{7}$ en esas comarcas. Hoy, definitivamente concluidas esas actividades extractivas, con criterios económicos y medioambientales esencialmente distintos de los que dimanaban de aquellas coyunturas, los vestigios mineros e industriales que nos han llegado generan paisajes culturales singulares que precisan ser identificados y catalogados, siendo susceptibles de ser, en una buena medida, conservados y puestos en valor.

Este proceso histórico-patrimonial se idea con una finalidad concreta: servir para su explotación sostenible desde la comunidad con el objetivo de producir -además de efectos culturales y sociales dotados de base histórica pasando a formar parte del acervo comunitario- recursos para activar económicamente zonas especialmente deprimidas, despobladas y con difíciles condiciones para su futuro. Al utilizar la sostenibilidad como eje para la explotación, bajo el concepto de Recurso de Uso Común (RUC) (Healy, 1994, citado en Calvo, Vega y Vázquez, 2020, 1), se configuran algunas características básicas de esos recursos como su no excluibilidad, lo que implica que no es posible excluir de su utilización a un usuario adicional o bien es muy costoso y que debe emplearse la totalidad de los recursos del área en el que se realiza la actividad lo que supone la convivencia de los visitantes con los habitantes del territorios. Esta configuración conceptual los convierte en bienes comunes complejos caracterizados por «múltiples, superpuestos, y potencialmente conflictivos usos y grupos de usuarios; volátiles en sus usos $\mathrm{y}$ acuerdos institucionales; y con variaciones entre los derechos de propiedad de jure y de facto" (Selsky \& Memon (2000), citado en Briassouris (2002) y en Calvo, Vega y Vázquez, 2020, 1).

Concretando nuestro discurso, estamos hablando de utilizar como RUC un conjunto de vestigios conformados por elementos singulares e individualmente relevantes: galerías y pozos mineros, castilletes para acceder al subsuelo, salas de máquinas, lavaderos y clasificadores del mineral, instalaciones para los mineros, dependencias administrativas y de gestión, centros asistenciales y de abastecimiento, infraestructuras viarias para ferrocarriles, vehículos, líneas de baldes, paisajes singulares derivados de explotaciones a cielo abierto, poblados y agrupaciones de viviendas, o servicios como economatos u hospitales. A todo ello se suma, por un lado, la documentación histórica, tanto los técnicos referidos a los edificios, explotaciones e instalaciones como los sociales, económicos etc... propios de empresas e instituciones relacionados con la actividad y la propia memoria de las gentes, de los habitantes, de los trabajadores, memoria colectiva imprescindible en un proyecto que tiene en la acción del hombre, su esencia. En definitiva, vestigios

7. El 16 de noviembre de 2018 se produjo el cierre de la última mina de carbón en la provincia de León. https://www.leonoticias.com/mineria/ultimo-mina-20181116130542-nt.html (Consultado el 08/04/2020). 
FRANCISCO M. BALADO INSUNZA

LA SENDA MINERA O CÓMO CONECTAR HISTORIA, PATRIMONIO Y DESARROLLO RURAL

de una actividad productiva y un proceso tecnológico de gran relevancia, con una absoluta influencia sobre el medio físico, social y económico en el que se produjo quedando como testigos directos y observables en un territorio delimitado: El Bierzo y Laciana.

Su valor, en el sentido hasta aquí apuntado, es incuestionable, pero debe ser identificado individualmente porque cada bien, cada referencia adquiere su propia personalidad, es relevante en su contexto local y, a la vez, lo trasciende formando parte de un conjunto patrimonial que se pretende valorizar porque conserva el carácter representativo de la historia más reciente de estos territorios y se imbrica en su acervo cultural y, por ende, forma parte sustancial de su memoria comunitaria.

Argumentos como los esbozados resultan la base de su consideración como recurso patrimonial con proyección económica para su explotación sostenible con la implicación de agentes públicos y privados. Y es así, con esta base en la que este conjunto de conceptos, conformadores de un paisaje cultural muy específico, sumatorio de elementos patrimoniales singulares surgidos como consecuencia del fin de la actividad minera e industrial en las comarcas mineras del oeste leonés es el contexto geográfico, cultural, patrimonial y económico en el que se plantea el proyecto La Senda Minera.

\section{LA SENDA MiNERA}

La Senda Minera (ver Fig. 1), es un proyecto del conservación, difusión y puesta en valor del patrimonio minero e industrial del oeste leonés: El Bierzo minero, Laciana y la Cepeda minera y ferroviaria que nace bajo el amparo ya indicado de la Cátedra de Turismo Sostenible y Desarrollo Local UNED-ULE, (CTS y DL). Este proyecto integrador y catalizador de la CTS y DL pretende identificar recursos, generar conocimiento y transferirlo a la sociedad con ideas viables económica y medioambientalmente, con un claro compromiso de establecer puntos de encuentro a partir de ideas que puedan ir plasmándose en proyectos innovadores que ayuden al desarrollo local ${ }^{8}$.

Los objetivos del Consorcio UNED Ponferrada y de la CTSDL, en colaboración con sus patronos: Ayuntamiento de Ponferrada, Diputación Provincial de León, Consejo Comarcal del Bierzo y Fundación Ciudad de la Energía, se centran en promover y desarrollar un proyecto para revitalizar turísticamente estas comarcas mineras, utilizando el patrimonio minero e industrial como recurso sostenible para el desarrollo local. Con los grandes recursos existentes se pretende crear productos asociados a ellos que permitan fijar población, generar actividad y crear empleo estable.

8. http://catedraturismosostenible.es/Consultada el 03/05/2020. 


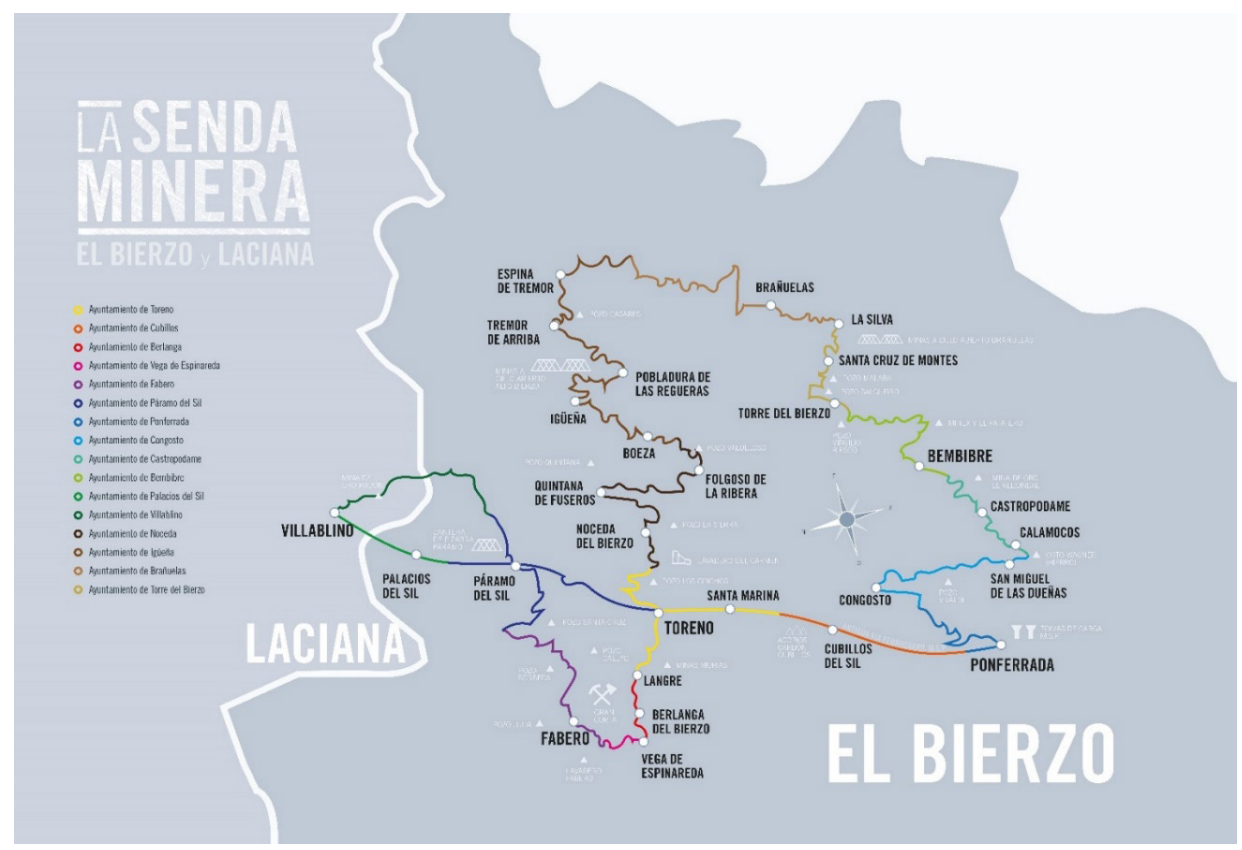

Figura n. ${ }^{\circ} 1$ Mapa inicial del proyecto La Senda Minera. Enero 2020. Elaboración propia.

Este patrimonio se presenta como un valor integral del territorio, un nexo entre los diferentes paisajes originados que, uniendo lo industrial y lo natural, se identifican en El Bierzo, en Laciana y en La Cepeda, pudiendo establecerse puntos de contacto que pueden conformar un discurso coherente con una sólida base histórica, cultural y, sobre todo, en términos actuales, integrador desde una perspectiva social y económica, de todo el espacio delimitado por la actividad minera de la zona.

La idea base de La Senda Minera consistiría en crear rutas accesibles que, con carácter inicialmente local, unan diferentes municipios a través de los recursos patrimoniales mineros e industriales de cada localidad o núcleo y, a su vez, unan municipios entre sí, generando una red/ruta/senda que cubra todo el territorio minero de El Bierzo, extendiéndose a Laciana y a la parte más occidental de La Cepeda y en el que, identificados los recursos de cada lugar, se puedan poner en valor y, paralelamente, se vayan organizando servicios e instalando dotaciones públicas que permitan atraer iniciativa privada de modo que se vaya generando un espacio de disfrute sostenible del conjunto patrimonial por la ciudadanía que vive en el territorio y por los visitantes que se reciban en los términos ya indicados en el anterior ítem de este trabajo. 
La Senda Minera, en términos generales, no se agotaría, sin embargo, en los aspectos turísticos o económicos, siendo éstos el primer objeto del proyecto y su gran reto, sino que la iniciativa pública lo hace especialmente interesante para que puedan identificarse otros elementos esenciales que la actividad minera e industrial nos ha dejado y que podrían ir sumando al producto global. Así, el segundo gran reto del proyecto, sería trasladar al proyecto la metodología general multidisciplinar que incorpore la memoria de las gentes que vivieron ese tiempo en estas comarcas, la de las empresas que se constituyeron, los movimientos sociales que se formaron, los paisajes que se transformaron... todo un complejo entramado que también forma parte de lo que hoy denominamos, en plural, patrimonios y que se pueden adjetivar, para individualizarlos, como patrimonio inmaterial, etnográfico, documental o natural.

Indicativamente resulta esencial que el proyecto dedique una parte de sus recursos a la recuperación, selección, catalogación y preservación del ingente patrimonio documental que nos ha dejado la actividad minera e industrial en estas comarcas mineras. Una parte importante ya se ha perdido, pero el resto debe ser objeto de atención preferente por su valor desde las diversas perspectivas que lo enfoquen.

Con estos principios y criterios se define el proyecto que requiere, necesariamente, la implicación de los agentes locales. Sin la participación de Juntas Vecinales, Ayuntamientos, Agentes y Grupos de Acción y Desarrollo Local, sociedad civil, en general, La Senda Minera es inviable.

Sin ánimo exhaustivo, recogemos elementos susceptibles de ser catalogados como patrimoniales en los diecisiete municipios propuestos inicialmente para participar en La Senda Minera.

\begin{tabular}{|l|l|}
\hline \multicolumn{1}{|c|}{ MUNICIPIO } & \multicolumn{1}{|c|}{ VESTIGIO PATRIMONIAL } \\
\hline CONGOSTO & INSTALACIONES COTO VIVALDI \\
& INSTALACIONES-CARGADEROS COTO WAGNER \\
& BOCAMINA VIVALDI \\
& ESTRUCTURAS METALICAS DE BALDES \\
& PUESTA EN VALOR RESTO DE VESTIGIOS MINERO- \\
& INDUSTRIALES MUNICIPIO \\
\hline CASTROPODAME & INSTALACIONES COTO WAGNER \\
& PUESTA EN VALOR RESTO VESTIGIOS MINERO- \\
& INDUSTRIALES MUNICIPIO \\
\hline BEMBIBRE & REVALORIZACFION BARRIO SOCUELLO \\
& PUESTA EN VALOR RESTO VESTIGIOS MINERO- \\
& INDUSTRIALES MUNICIPIO \\
\hline
\end{tabular}


FRANCISCO M. BALADO INSUNZA

LA SENDA MINERA O CÓMO CONECTAR HISTORIA, PATRIMONIO Y DESARROLLO RURAL

\begin{tabular}{|c|c|}
\hline MUNICIPIO & VESTIGIO PATRIMONIAL \\
\hline TORRE DEL BIERZO & $\begin{array}{l}\text { ESTACION FERROCARRIL TORRE DEL BIERZO } \\
\text { POZO VILORIA } \\
\text { MINA DE ORO } \\
\text { POZO SALGUEIRO } \\
\text { PUESTA EN VALOR RESTO VESTIGIOS MINERO- } \\
\text { INDUSTRIALES MUNICIPIO }\end{array}$ \\
\hline BRAÑUELAS-VILLAGATON & $\begin{array}{l}\text { MINA MALABÁ } \\
\text { ESTACION FERROCARRIL BRAÑUELAS } \\
\text { PUESTA EN VALOR RESTO VESTIGIOS MINERO- } \\
\text { INDUSTRIALES MUNICIPIO }\end{array}$ \\
\hline IGUEÑA & $\begin{array}{l}\text { POZO CASARES } \\
\text { PUESTA EN VALOR DE LOS VESTIGIOS MINERO- } \\
\text { INDUSTRIALES MUNICIPIO }\end{array}$ \\
\hline NOCEDA & $\begin{array}{l}\text { PUESTA EN VALOR VESTIGIOS MINERO- } \\
\text { INDUSTRIALES MUNICIPIO }\end{array}$ \\
\hline FOLGOSO DE LA RIBERA & $\begin{array}{l}\text { MINA ESCUELA } \\
\text { PUESTA EN VALOR VESTIGIOS MINERO- } \\
\text { INDUSTRIALES MUNICIPIO }\end{array}$ \\
\hline TORENO & $\begin{array}{l}\text { TRAMO FERROCARRIL PV } \\
\text { ESTACION PV } \\
\text { INSTALACIONES ANTRACITAS DE GAIZTARRO } \\
\text { PUESTA EN VALOR RESTO VESTIGIOS MINERO- } \\
\text { INDUSTRIALES MUNICIPIO }\end{array}$ \\
\hline PARAMO DEL SIL & $\begin{array}{l}\text { TRAMO FERROCARRIL PV } \\
\text { ESTACION PV } \\
\text { LAVADERO LA RECUELGA } \\
\text { POBLADO SANTA CRUZ DEL SIL } \\
\text { EL ESCOBIO } \\
\text { PUESTA EN VALOR VESTIGIOS MINERO- } \\
\text { INDUSTRIALES MUNICIPIO }\end{array}$ \\
\hline PALACIOS DEL SIL & $\begin{array}{l}\text { TRAMO FERROCARRIL PV } \\
\text { ESTACION PV } \\
\text { APEADERO CORBON } \\
\text { PUESTA EN VALOR RESTO VESTIGIOS MINERO- } \\
\text { INDUSTRIALES MUNICIPIO }\end{array}$ \\
\hline
\end{tabular}


FRANCISCO M. BALADO INSUNZA

LA SENDA MINERA O CÓMO CONECTAR HISTORIA, PATRIMONIO Y DESARROLLO RURAL

\begin{tabular}{|c|c|}
\hline MUNICIPIO & VESTIGIO PATRIMONIAL \\
\hline VILLABLINO & $\begin{array}{l}\text { TRAMO FERROCARRIL PV } \\
\text { ESTACION PV } \\
\text { POZO MARIA } \\
\text { POZO CALDERON } \\
\text { LAVADERO MSP } \\
\text { PUESTA EN VALOR RESTO VESTIGIOS MINERO- } \\
\text { INDUSTRIALES MUNICIPIO }\end{array}$ \\
\hline FABERO & $\begin{array}{l}\text { CONJUNTO URBANO.MINERO } \\
\text { POZO JULIA } \\
\text { POZO VIEJO } \\
\text { POZO ALICIA } \\
\text { POBLADO DIEGO PEREZ } \\
\text { ESTRUCTIURAS METALICAS DE LA LINEA DE BALDES } \\
\text { LA GRAN CORTA } \\
\text { PUESTA EN VALOR VESTIGIOS MINERO- } \\
\text { INDUSTRIALES MUNICIPIO }\end{array}$ \\
\hline VEGA DE ESPINAREDA & $\begin{array}{l}\text { PUESTA EN VALOR VESTIGIOS PATRIMONIALES E } \\
\text { HISTORICOS DEL MUNICIPIO }\end{array}$ \\
\hline BERLANGA DEL BIERZO & $\begin{array}{l}\text { LA GRAN CORTA } \\
\text { PUESTA EN VALOR VESTIGIOS MINERO- } \\
\text { INDUSTRIALES MUNICIPIO }\end{array}$ \\
\hline CUBILLOS DEL SIL & $\begin{array}{l}\text { COMPOSTILLA II } \\
\text { TRAMO FERROCARRIL PV } \\
\text { ESTACION PV } \\
\text { PUESTA EN VALOR VESTIGIOS MINERO- } \\
\text { INDUSTRIALES MUNICIPIO }\end{array}$ \\
\hline PONFERRADA & $\begin{array}{l}\text { COMPOSTILLA I } \\
\text { POBLADO ENDESA } \\
\text { LA FABRICA DE LUZ } \\
\text { POBLADO MSP } \\
\text { MUSEO DEL FERROCARRIL } \\
\text { LA PLACA } \\
\text { VESTIGIOS MONTAÑA DE CARBON } \\
\text { FUENTE DEL AZUFRE } \\
\text { MINAS DE WOLFRAMIO } \\
\text { ANTIGUO MATADERO } \\
\text { POBLADO MONTEARENAS } \\
\text { PUESTA EN VALOR OTROS VESTIGIOS MINERO- } \\
\text { INDUSTRIALES MUNICIPIO }\end{array}$ \\
\hline
\end{tabular}

Figura n. ${ }^{\circ} 2$ Relación indicativa de recursos patrimoniales mineros. Elaboración propia. 


\section{El modelo de Gestión de La Senda Minera}

Los ejes fundamentales con los que se presenta este proyecto de desarrollo local se sustentan en un modelo de gestión público-privado muy específico, que pretende crear un recurso turístico de calidad de base patrimonial, que imbrique a todas las partes en su puesta en práctica, al estar enraizado en la propia identidad histórica y cotidiana de la comunidad donde se expresa, de modo que lo hagan propio y cada una, en la medida de su participación, coadyuven a su mantenimiento en el tiempo.

Cada actor local debe identificar los bienes que, en su ámbito geográfico propio, pueden incorporarse a la Senda. Una explotación minera, una infraestructura industrial, maquinaria utilizada en la extracción o en el transporte, una vía de conexión, alguna estructura auxiliar..., elementos todos ellos que deben catalogarse, identificarse técnica y cualitativamente. Una labor previa e imprescindible para dotar de consistencia al proyecto.

Identificar, inventariar y catalogar no es tarea sencilla ni mucho menos, rápida. Requiere la presencia de conservadores del patrimonio, documentalistas, historiadores, arquitectos, profesionales, en definitiva, que, con criterios estrictamente técnicos, van a determinar esta primera parte del proceso, su valor y alcance. No todo es conservable, por distintas razones.

La calificación y la valoración de los vestigios de las explotaciones como integrantes del patrimonio minero e industrial comportan habitualmente una fuerte carga de subjetividad y, además, no es fácil separar los criterios de identificación de los de valoración. La identificación de un elemento ya comporta una valoración, a priori. Atribuir valor patrimonial a una determinada explotación minera puede ser fruto de una opinión generalizada, de un grupo de ámbito regional o local, o de un investigador independiente (PEARSON \& MCGOWAN, 2000). De ahí nuestra postura de acometer este trabajo desde una perspectiva estrictamente técnica con un equipo multidisciplinar como el indicado que sea el que, en primer lugar, identifique los vestigios a considerar, paso imprescindible a modo de inventario con el que poder avanzar, en un segundo momento, hacia la valoración o cuantificación del interés que ese bien tenga para ser conservado.

Este proceso es esencial para la patrimonialización de estos vestigios. Su conversión en recursos con proyección turística que sirvan de base para un producto atractivo para el visitante debe nacer en su correcta y precisa identificación, el conocimiento de su evolución histórica, una descripción de usos y su valor a efectos de ser reconocido y, finalmente, conservado como un elemento relevante para ser exhibido hacia el exterior, como integrante parte del acervo cultural, patrimonial del lugar.

Esta labor proactiva, en primer lugar, interna, hacia la propia comunidad que lo identifica como bien patrimonial, lo que requiere una labor educativa imprescindible y, posteriormente, hacia el exterior, ya como expresión del patrimonio propio que define a la comunidad y que se ofrece a terceros, generando un valor 
con consecuencias económicas, es decir, con retorno hacia la propia colectividad que lo ha reconocido, conservado y puesto en valor, ahora en términos económicos, ya individuales, con acciones fundamentadas en discursos basados en ese patrimonio comunitario, ya colectivas, como indicación de los atractivos de la localidad concreta que los incluye en su oferta turística.

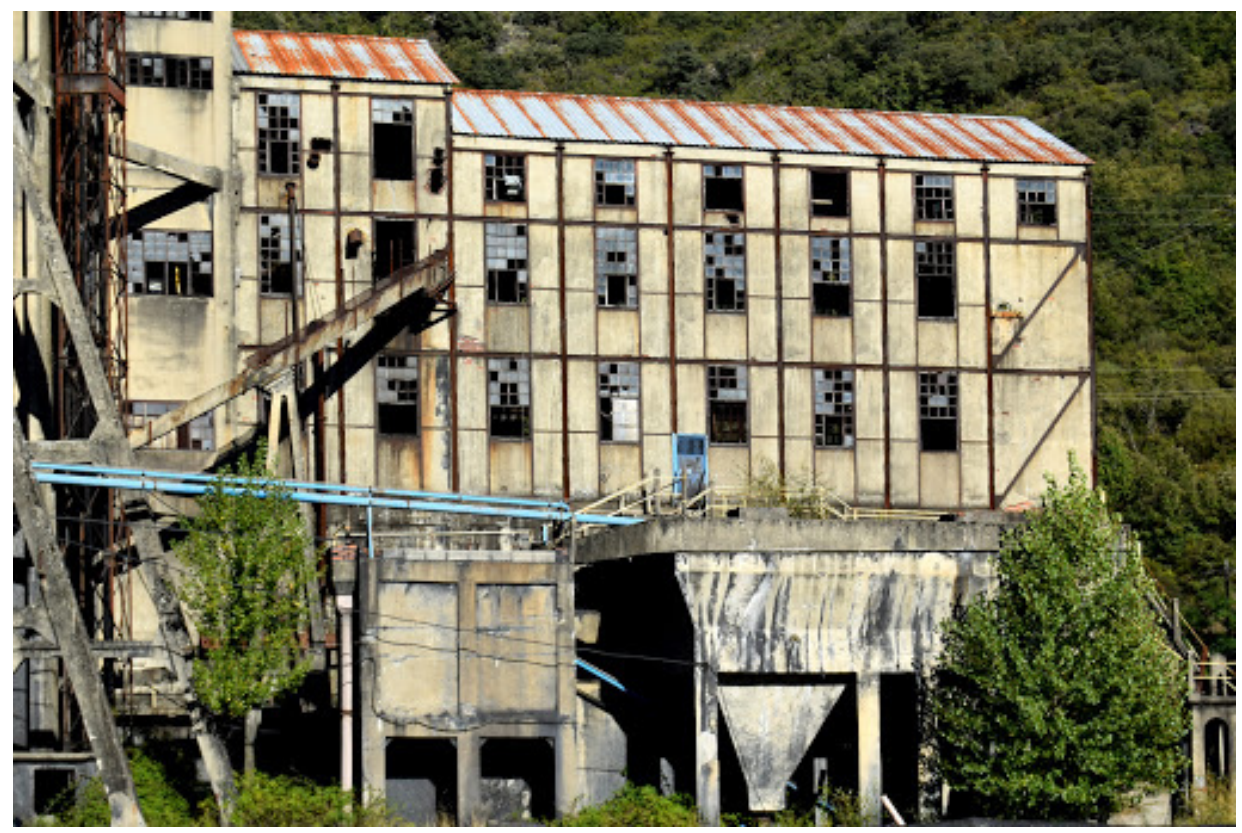

Foto n. ${ }^{\circ}$ 1. 1 Lavadero de La Recuelga. Santa Cruz del Sil. Estado Actual.

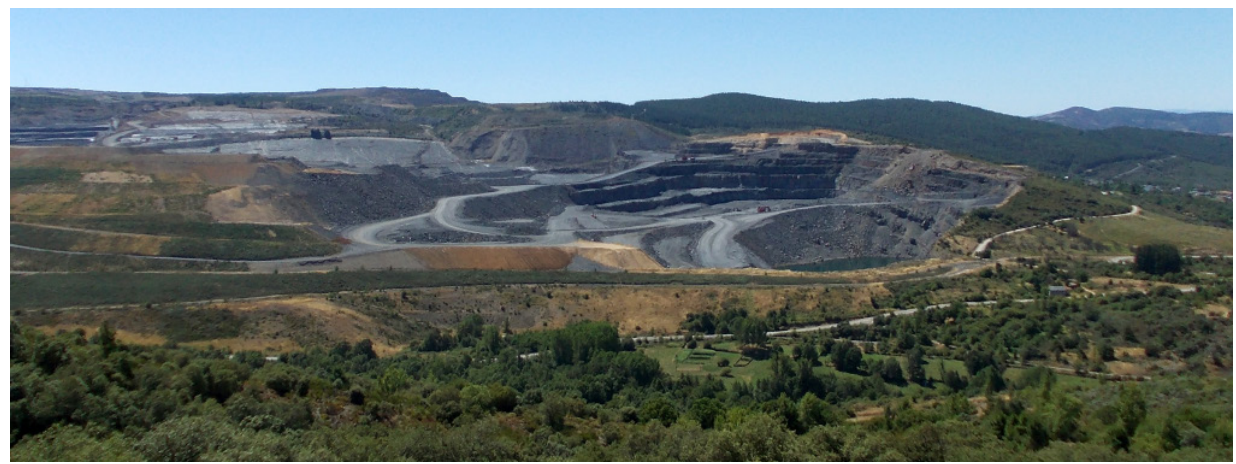

Foto n. ${ }^{\circ}$ 2. Gran Corta de Fabero. http://www.archivohistoricominero.org/portfoliocategory/gran-corta-de-fabero-fabero-del-bierzo-leon/ 


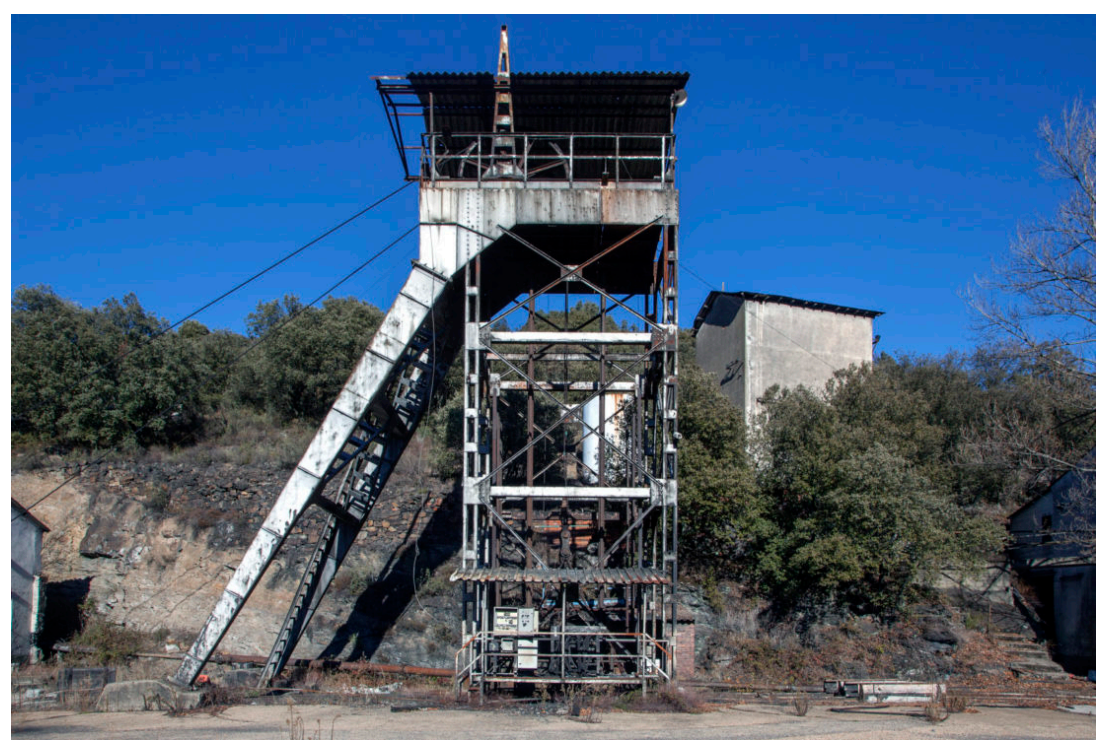

Foto n. ${ }^{\circ}$ 3. Castillete del Pozo Malabá. La Silva. Foto. Francisco M. Balado.

La labor o proceso de identificación, reconocimiento, descripción de calidad y valoración de los recursos patrimoniales de cada localidad o territorio concreto, como los que recogemos en las fotografías 1, 2, 3 y 4, a modo de ejemplo, que puedan ser incluidos en La Senda Minera, aplicando criterios tales como el estado de conservación, su importancia histórica, la ubicación estratégica, su relevancia individual... resulta una ardua pero imprescindible acción. Es la primera que debe acometerse y, por razones de economía, en el propio cronograma del proyecto debe realizarse de forma paralela a la definición de un proceso de análisis y desarrollo de un modelo de gestión integrada del proyecto, labor que incumbe a todos los agentes con capacidad para ello que operan en el territorio de manera que, bajo unos principios generales como los indicados: recuperación, preservación, sostenibilidad e innovación, recoja la acción pública dirigida a generar y transferir conocimiento, la orientada a sentar las bases de intervención y coordinación entre las distintas administraciones y la específicamente dirigida a la gestión de un plan acción integral de modo que, bajo la coordinación de un mecanismo ágil, dote al territorio de infraestructuras y recursos que sean susceptibles de establecer condiciones suficientemente atractivas para que la iniciativa privada pueda generar actividad económica vinculada a estos recursos, cree empleo de calidad y pueda ayudar a fijar población en el medio rural, fundamentalmente.

En esta línea, la propuesta se presenta como algo de mayor complejidad y, por consiguiente, precisa dotarse de instrumentos que incluyan la participación desde abajo (agentes locales: municipios, pedanías, Grupos de Acción Local) y su 
coordinación con gestores y agentes públicos con capacidad financiera y organizativa (Fundación Ciudad de la Energía y/o Administraciones Públicas de ámbito superior al municipal). Ver Fig. 3.

Será el conjunto el que, dando continuidad a las líneas de actuación del Consorcio Universitario UNED-CTS y DL, explore la oportunidad que se presenta generando un grupo inicial de trabajo que, sin preestablecer formas jurídicas que serían objeto de debate e implementación consensuada, desarrolle el proyecto y que podría formarse entre las Administraciones y Organismos Públicos implicados y el Consorcio Universitario UNED-CTS y DL, aportando las partes su experiencia y recursos, sin exclusividades y al que se podrían incorporar, como instrumentos político-administrativos de referencia en el territorio, la Diputación Provincial de León o el Consejo Comarcal del Bierzo, entes aglutinadores de los diferentes órganos locales y vecinales de ámbito territorial inferior.

\section{PLANO PUBLICO}

\section{CONSORCIO PUBLICO LA SENDA MINERA}

DIPUTACION PROVINCIAL DE LEON / CONSEJO COMARCAL DEL BIERZO

\section{AYUNTAMIENTOS Y JUNTAS VECINALES}

CONSORCIO PUBLICO UNED - CTS yDL

FUNDACION CIUDAD DE LA ENERGIA

PLANO PRIVADO

EMPRENDEDORES Y EMPRESAS

Figura n. ${ }^{\circ}$ 3. Modelo de gestión público-privada de La Senda Minera.

El esquema, inicialmente doble y horizontal, se iría conformando con la aportación de cada entidad sumando activos de cada una de ellas, con una metodología, inicialmente prospectiva, posteriormente analítica y finalmente, ejecutiva, que permita identificar un modelo integrador social y territorialmente capaz de poner en marcha un plan de acción en los términos esbozados.

Como se muestra en el esquema, se propone una gestión inicialmente pública con un Consorcio formado por los municipios integrados en la Senda Minera y la Administración supramunicipal que sea la referencia. El Consorcio Público 
UNED Ponferrada, a través de la CTS y DL como servicio de estudios y oficina de proyectos podría incorporarse, en los términos que se concreten, a la gestión del proyecto.

Finalmente, la implementación de recursos turísticos y de ocio permitirán, en el medio plazo, la construcción de un sistema en red en los distintos municipios con recursos como aulas geológicas, tirolinas, ciclo-railes, campings, albergues turísticas, visitas virtuales a través de realidad aumentada, planetario, actividades acuáticas, puentes tibetanos, pasarelas, centros de desarrollo BTT, Alpin Coster, parques infantiles, Parque de los sentidos mineros, visitas a minas de interior, cabañas aéreas, concursos nacionales sobre turismo industrial creativo, rutas y centros culturales, mini estaciones, parques temáticos, puesta en valor del Ponfeblino, de la Mina Escuela de Folgoso, del Museo del Ferrocarril de Brañuelas, del Museo de la Fundación Cultura Minera de Torre del Bierzo, participación en proyectos de desarrollo de León Sostenible como INCUBA, el Centro de Innovación y Emprendimiento en el Territorio (CIET) de la CTS y DL, entre otros.

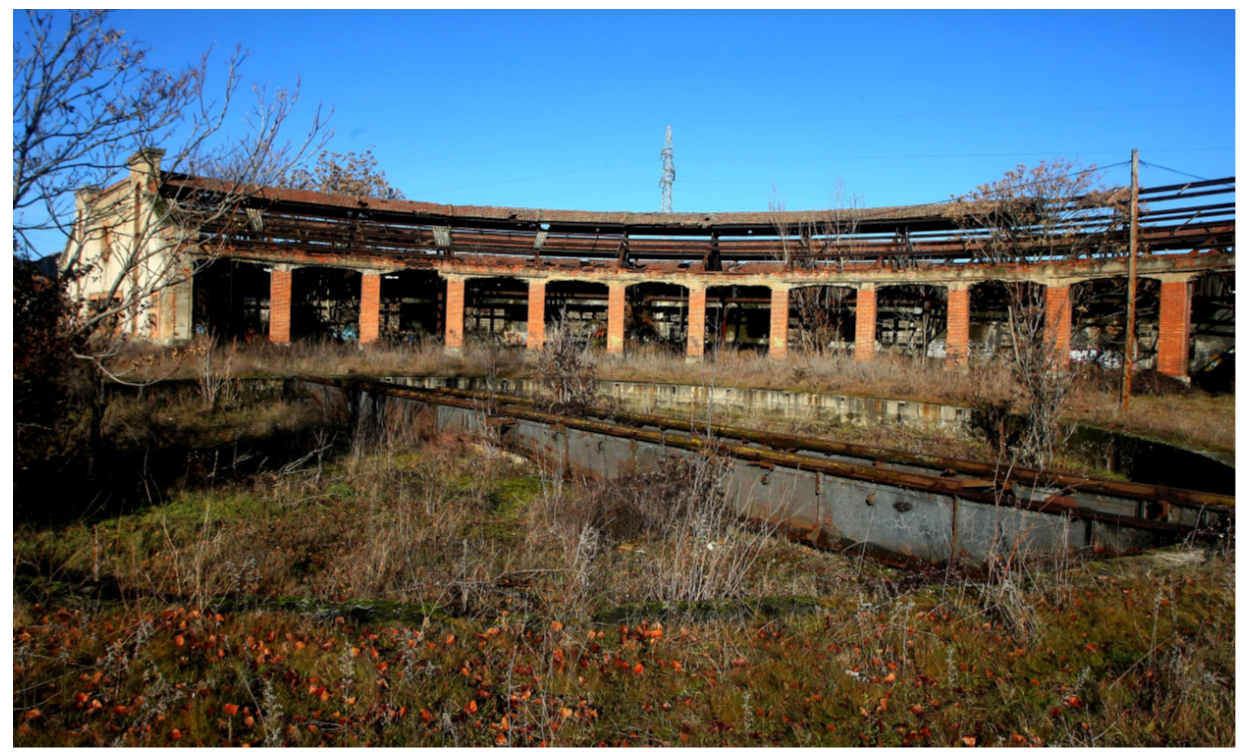

Foto n. ${ }^{\circ}$ 4. La Placa. Ponferrada. Foto: Francisco M. Balado.

\section{CONCLUSIONES}

Aunque el objeto de esta contribución es el de la presentación de un proyecto de dinamización territorial concreto, referido a las comarcas mineras del carbón y del hierro periféricas de Castilla y León, El Bierzo y Laciana, y no tanto 
la construcción científica de un armazón que lo justifique teóricamente, si es necesario concluir algunas de las reflexiones hasta ahora formuladas.

Así, en cuanto a los principios generales del proyecto, se pretende identificar elementos esenciales que la actividad minera e industrial ha dejado en una zona territorial delimitada, sin perjuicio de poder escalar y replicar el modelo en otros espacios y referidos $\mathrm{s}$ otros recursos, y que podrían ir sumando a un proyecto colaborativo que, desde los ámbitos locales, conformen un producto de desarrollo económico territorial global.

De igual modo, La Senda Minera se configura como una ruta que conecte los municipios mineros del carbón y del hierro de las comarcas indicadas, partiendo de la identificación de sus recursos patrimoniales individuales, lo que subraya sus singularidades para, de modo sumatorio, ir uniéndolos geográficamente (Ver Fig. 2) y formar una red/senda que conecte todos los polos, complementariamente entre sí.

Las rutas así planteadas, inicialmente de carácter local y municipal, forman un proyecto unitario al ensamblarse diferentes recursos de cada enclave local. Esta unión de recursos conecta, también, municipios entre sí, construyendo una ruta que cubre todo el territorio minero de El Bierzo y Laciana.

Para generar esta ruta/senda se precisa una inversión paralela en servicios y dotaciones públicas que delimitan un espacio de disfrute sostenible del conjunto patrimonial por la ciudadanía que vive en el territorio y por los visitantes que se reciban.

Esta configuración de abajo hacia arriba y de base pública debe contribuir de manera decisiva a la creación de las condiciones para atraer iniciativa privada. El fomento del emprendimiento es, finalmente, la clave del desarrollo rural porque la generación de actividad económica vinculada a estos recursos patrimoniales capaz de crear empleo de calidad y ayudar a fijar población en el medio rural es decisiva.

En cuanto al modelo de gestión, se pretende implementar un modelo público-privado de gestión de tipo horizontal, ágil, participativo, riguroso y transparente. Un modelo que sea sostenible económica, medioambiental y socialmente basado en la recuperación y preservación patrimonial.

La gestión de un proyecto de este tipo, innovador social y económicamente, colaborativo, abierto y transparente tiene como base la instalación de elementos de innovación tecnológica que sitúen la gestión en el campo de la conservación preventiva del patrimonio minero e industrial. Un modelo preventivo incorpora el análisis de las amenazas y riesgos, su monitorización y su conexión con los sistemas públicos de información preventiva y de señalización.

En definitiva, un proyecto que se destina a incentivar el emprendimiento, la fijación de población, la generación de empleo estable y, por ende, el desarrollo local. Un ejemplo de ello es el Museo la Fábrica de Luz en Ponferrada (Foto 5), paradigma de la valorización y preservación del patrimonio industrial derivado de la actividad extractiva y que constituye uno de los hitos que se incorporan a una ruta como la que constituye La Senda Minera. 


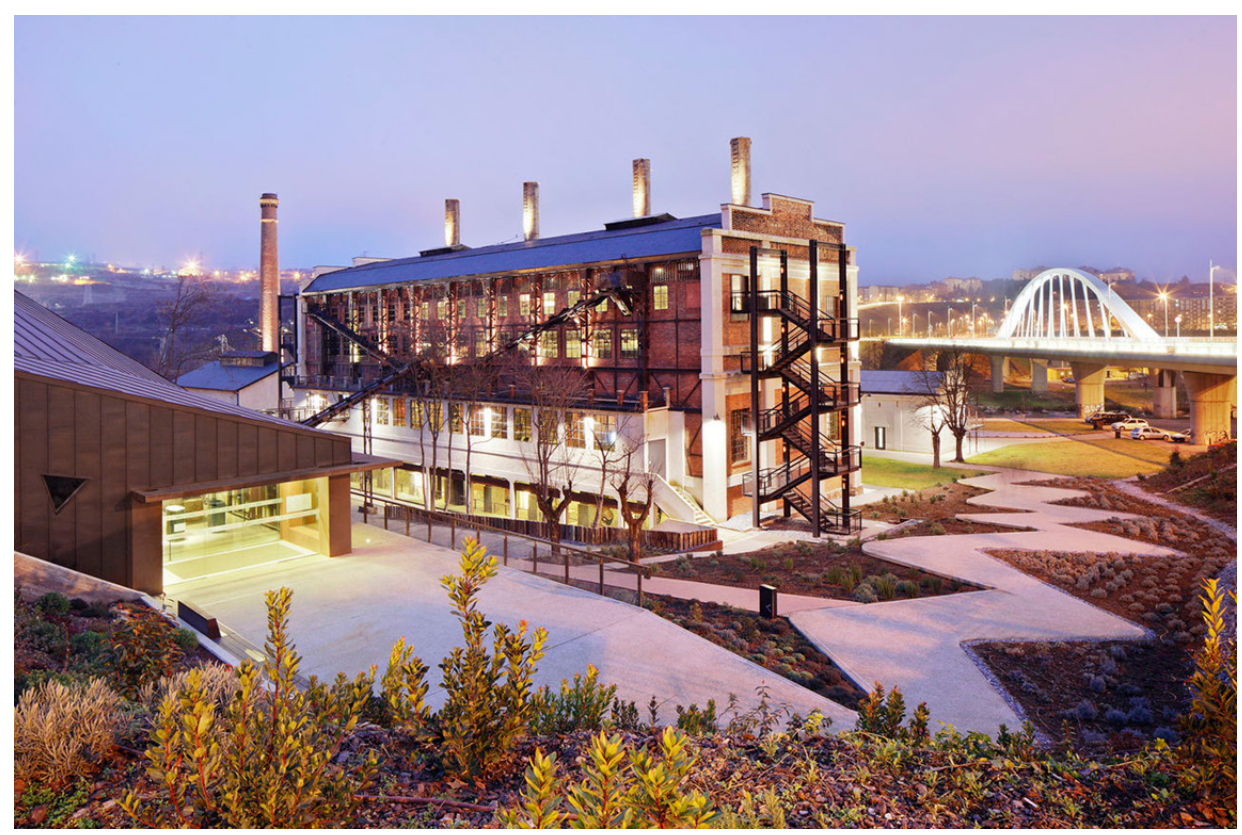

Foto n. ${ }^{\circ}$ 5. Museo de la Energía. Fábrica de Luz. Ponferrada.

\section{REFERENCIAS BIBLIOGRÁFICAS}

Balado Insunza, F. M. (2018). 1918: el comienzo del corto siglo XX en el Bierzo, Estudios Bercianos, 41, pp. 17-35.

Briassuolis, H. (2002). Sustainable Tourism and the Question of the Commons. Annals of Tourism Research, 29(4) pp. 1065-1085.

Calvo, J. L., Vega. J y Vázquez Burguete, J. L. (2020). Turismo sostenible. un enfoque de bienes comunes y economía del donut. Sustainable Tourism. a Common Goods and Doughnuts Economy Approach. Paper Presented 19a Iapnm, Ponferrada, León, España.

Calvo, J. L., Vega. J y Vázquez Burguete, J. L. (2020). La cátedra de turismo sostenible y desarrollo local. En G. Cavero (coord.), Actas del congreso del MC aniversario del Monasterio de San Pedro de Montes (919-2019), isbn: 978-84-18490-03-3, pp. 303-308.

Féraud, J., Martins, L., Philippon, J. y Barge, H. (2001). Les impératifs de valorisation économique et touristique du patrimoine representé par d'anciens sites miniers: le rôle des services géologiques européens auprés des archéologues. Congresso Internacional sobre Patrimonio Geológico e Mineiro. Beja (Portugal).

Forcadell Álvarez, C. (1996). De la revolución democrática a la restauración: el horizonte de una historia socia. En M. Esteban de Vega y A. Morales Moya (coords), La historia contemporánea en España: Primer Congreso de Historia Contemporánea de España. Salamanca, 1992, pp. 103-104. 
Mazadiego Martínez, L. F. (2003). Folclore, leyendas y costumbres mineras: el patrimonio inmaterial de la minería. Utrillas, Teruel.

Puche Riart, O., García Cortés, A. y Mata Perelló, J. M. (1994). Conservación del patrimonio bistórico Minero-metalúrgico Español. León.

Roldan, S., García Delgado, J. L. (eds.). (1973). La formación de la sociedad capitalista en España 1914-1920 (II tomos). Madrid: Ceca.

Rubio, A. (2012). Introducción histórica. espacios al servicio de la actividad económica. En La minería del carbón en Castilla y León desde el punto de vista de su sostenibilidad y de su carácter como reserva estratégica. CES, Castilla y León, 201, pp. 57-82.

Selsky, J. y Memon, P. (2000). Emergent commons: local responses in complex CPR systems. Paper presented at the $8^{\text {th }}$. IASCP Conference, Bloomington, Indiana. 
